\section{EL IMPACTO DE LA INCERTIDUMBRE EN LA TRANSICIÓN A LA EDAD ADULTA: POSICIONES BIOGRÁFICAS Y CONSECUENCIAS SOBRE LA VIDA}

\author{
Izaskun Artegui Alcaide \\ Universidad del País Vasco/Euskal Herriko Unibertsitatea \\ izaskun.artegui@ehu.eus \\ ORCID iD: http://orcid.org/0000-0002-4091-5446
}

\section{THE IMPACT OF UNCERTAINTY IN THE TRANSITION TO ADULTHOOD: BIOGRAPHIC POSITIONS AND CONSEQUENCES ON LIFE}

Cómo citar este artículo/Citation: Artegui Alcaide, I. (2017). El impacto de la incertidumbre en la transición a la edad adulta: Posiciones biográficas y consecuencias sobre la vida. Arbor, 193 (784): a379. doi: http://dx.doi.org/10.3989/arbor.2017.784n2002

Recibido: 17 junio 2014. Aceptado: 9 mayo 2016.

RESUMEN: Las transformaciones del mercado laboral han provocado la diversificación de los tiempos de trabajo. Las pautas temporales que guiaban el transcurso vital se erosionan: la incertidumbre se instala en las vidas. Los jóvenes que se encuentran en la última etapa de la transición a la edad adulta sufren con especial crudeza esta situación. Las expectativas que construyeron en el pasado chocan con su presente. A través del análisis de veinticuatro entrevistas en profundidad, estudiamos de qué manera impacta la incertidumbre sobre estos jóvenes y qué efectos tiene sobre sus vidas. Los datos producidos en el trabajo de campo nos muestran que tanto el nivel de impacto, como las consecuencias de la incertidumbre, son diferentes en los jóvenes que siguen la secuencia del ciclo vital estandarizado y en quienes la han abandonado.

PALABRAS CLAVE: Transición de la juventud a la edad adulta; ciclo vital; incertidumbre; expectativas de futuro; inestabilidad y precariedad laboral.
Copyright: (c) 2017 CSIC. Este es un artículo de acceso abierto distribuido bajo los términos de la licencia Creative Commons Attribution (CC BY) España 3.0.
ABSTRACT: The labor market's transformations have led to the diversification of working time. The temporal patterns that guided the life course are eroding: uncertainty is installed in the lives. Young people who are in the last stage of the transition from youth to adulthood are suffering this reality particularly hard. The expectations that they built in the past clash with their present. Through the analysis of twenty-four in-depth interviews we analyze how uncertainty impacts on young people's lives and what effects it has on them. The data produced in the fieldwork show us that both the level of impact, as the consequences of uncertainty, are different for young people in the standardized lifecycle sequence and for those who have dropped it out.

KEYWORDS: Transition from youth to adulthood; lifecycle; uncertainty; future expectations; labor precariousness and instability. 


\section{INTRODUCCIÓN}

La secuencia de etapas que completa el ciclo vital, dice Luhmann (1976), conforma un mecanismo de simplificación que indica al individuo cuál será la siguiente etapa a la que se tendrá que enfrentar. Este dispositivo reduce tanto la apertura ligada al futuro como su complejidad, dando lugar a lo que el autor denomina como "desfuturización del futuro". El buen funcionamiento de esta maquinaria, no obstante, requiere de una estructura temporal normalizada y de unos contrafuertes lo suficientemente sólidos que la soporten. El modelo de ciclo vital estandarizado que impera en los imaginarios sociales actuales es fruto de la estructura temporal que surge de la combinación de dos elementos paradigmáticos de la sociedad industrial: el estado de bienestar y el sistema productivo fordista. Ambos elementos dieron lugar a la norma social de empleo de la época dorada del capitalismo (Alonso, 1999; Alonso, 2007; Prieto, 2007); norma social que, gracias a la expansión de un ideal de trabajo lineal, estable y protegido, permitió a una parte sustancial de la sociedad desarrollar unos planes de vida sujetos a esas mismas premisas de linealidad, estabilidad y predictibilidad. Es pertinente señalar, no obstante, que esta norma, habitualmente generalizada tanto en los trabajos académicos como en el imaginario social, se ha limitado a recoger en su definición las características propias de un tipo de trabajador concreto: el trabajador industrial y varón, dejando fuera de ella otras realidades extensas, como la de las mujeres que, atendiendo a sus condiciones de trabajo ya desde la época que señalamos, ha generado una "norma del empleo" mucho más cercana a la discontinuidad, a la inestabilidad y a la precariedad que la generalizada en el popular modelo masculino (Carrasquer y Torns, 2007; Torns y Recio Cáceres, 2012).

En todo caso, la crisis que comienza en los años 70 ataca de forma radical a las instituciones que soportaban la norma social del empleo masculina y su popular premisa biográfica. Desde entonces hasta la actualidad, las continuas flexibilizaciones y desregulaciones que ha sufrido el mercado laboral han provocado una diversificación de los aspectos temporales del trabajo (Castro, 2008) que no solo pone en duda la posibilidad de continuar guiando las vidas con arreglo al modelo biográfico sujeto a la norma social del empleo masculino, sino que empuja, sobre todo a aquellos colectivos más vulnerables, a reproducir un modelo mucho más cercano a la norma femenina. Con estas transformaciones, la secuencia de etapas que guiaba el transcurso vital se diversifica. El futuro se abre y se complejiza. La estructura temporal normalizada sujeta a la norma social del empleo masculino se sustituye por una estructura temporal diversificada y compleja que hace difícil adivinar cuáles serán las características de la siguiente etapa o qué herramientas serán necesarias para afrontarla.

Este fenómeno no solo impacta sobre aquellos perfiles más vulnerables dentro del mercado laboral, sino también sobre aquellos colectivos que se encuentran en etapas de transición. En este artículo, pondremos la mirada sobre uno de ellos. Concretamente, sobre los jóvenes que ocupan la última fase de la transición de la juventud a la edad adulta. Socializados en la idea de un ciclo vital estandarizado con base en la norma de empleo masculina descrita, muchos jóvenes se topan hoy con una realidad biográfica ciertamente diversificada: los patrones sobre los que crearon sus expectativas pierden validez, sus trayectorias laborales se caracterizan por la inestabilidad, la precariedad y el desempleo, sus salarios son tan escasos como sus propios derechos laborales, sus transiciones se complican, sus planes de futuro se retrasan... (Cavalli, 1985; Côté, 2000; Gentile, 2014; Leccardi, 2002; Leccardi, 2008; Leccardi y Ruspini, 2006; Moreno Mínguez, López Peláez y Segado Sánchez-Cabezudo, 2012; Pais, 2003; Reiter, 2003; Santamaría, 2010; Santamaría, 2012; Santos y Martín, 2012). Estas cuestiones, en su conjunto, llenan sus futuros y sus vidas de una fuerte sensación de incertidumbre.

Partiendo de este contexto, el principal objetivo de este artículo es estudiar de qué manera impacta tal incertidumbre sobre las vidas de estos jóvenes y detectar cuáles pueden ser sus posibles efectos a largo plazo. Para ello, nuestra hipótesis de partida es que tanto el nivel de impacto de la incertidumbre como sus distintas consecuencias están condicionados por el grado de persistencia de la biografía normalizada en sus imaginarios. Para falsar esta hipótesis nos basaremos en los datos producidos a través de veinticuatro entrevistas en profundidad realizadas en 2013. A la hora de conformar la muestra, ponemos la atención, fundamentalmente, en dos grupos de variables. Por una parte, buscamos jóvenes emancipados o en vías de emancipación, con estudios finalizados al menos cuatro años antes de la realización de la entrevista y con cierta experiencia laboral posterior a la conclusión de sus estudios, de lo que resulta una horquilla de edad que oscila entre los veintisiete y los treinta y cinco años de edad. Con estas variables, tratamos de acceder a aquellas personas jóvenes económicamente independientes o semi-independientes que 
viven los primeros años del paso de lo formativo a lo laboral, etapa y circunstancias en las que se concentran los principales problemas de la transición de la juventud a la edad adulta. Por otra parte, nos dirigimos a jóvenes con estudios superiores y procedentes de familias de clase media o clase trabajadora. Estas dos variables nos permiten acercarnos a un perfil que ha sido socializado y busca reproducir con especial insistencia el modelo masculino de biografía fordista (lineal, estable y ascendente). Más concretamente, se trata de un grupo de jóvenes que, con el visto bueno de sus progenitores, ha prorrogado su situación de dependencia familiar en aras de una entrada al mundo adulto en unas condiciones de movilidad social ascendente (Bernardi, 2007). Ambas variables, constreñidas sociológicamente en edad y clase social, nos permitirán dirigirnos a un colectivo de jóvenes clave para los objetivos de la investigación: el análisis del choque de expectativas y su influencia en el manejo de la incertidumbre de futuro.

Además de estas variables, también atendemos al género y al perfil formativo de las personas entrevistadas. En lo que se refiere al género, se realizan doce entrevistas a mujeres y doce a hombres con el fin de averiguar de qué manera interviene el género de las personas jóvenes en sus trayectorias biográficas y en sus expectativas. En el caso de los estudios superiores, buscamos acceder a una variedad de perfiles amplia con el objetivo de detectar diferentes vivencias y experiencias tanto de inserción laboral como de calidad del empleo en los primeros años de trayectoria laboral (linealidad, precariedad, tipo de contratación, tiempo transcurrido desde la finalización de los estudios y primer trabajo...). Finalmente, cabe señalar que todos los jóvenes residían en la Comunidad Autónoma del País Vasco cuando se realizaron las entrevistas.

\section{LA DIVERSIFICACIÓN DE LOS TIEMPOS DE TRABAJO}

Como señalan Piore y Sabel en su obra de referencia La segunda ruptura industrial (1990), la crisis de los años 70 pone en evidencia el agotamiento del paradigma productivo, propiamente masculino ${ }^{1}$, de la etapa industrial. La búsqueda de un modelo productivo menos rígido y más moldeable frente a las constantes fluctuaciones del mercado allana la entrada al modelo de producción flexible que llega hasta nuestros días. Más allá de las consabidas flexibilizaciones que acarrea este modelo de producción (la flexibilización de las estructuras empresariales, de los procesos de producción, de los productos, de la localización...), nos interesa ahora centrarnos en un proceso de flexibilización puntual: la flexibilización de los tiempos de trabajo.

Castro (2008) en un interesante artículo sobre el papel de las expectativas en las estrategias de coordinación de los tiempos de trabajo, destaca cuatro aspectos temporales específicos a los que hay que atender en el análisis del tiempo laboral: el tiempo contractual, el tiempo de prestación laboral (esto es, los horarios y calendarios), el tiempo de la vida laboral y las expectativas laborales ${ }^{2}$. Desde el análisis específico de estos aspectos, el autor defiende que el asentamiento o la expansión del sistema de producción flexible ha derivado en una particular diversificación de cada uno de ellos. Nos interesan las conclusiones que extrae el autor respecto al análisis de la diversificación de dos de estos aspectos: las biografías laborales y las expectativas laborales. En lo que se refiere a la diversificación de las biografías laborales, el autor destaca dos efectos de la flexibilización de los tiempos de trabajo: el acortamiento de la trayectoria profesional y la ruptura de la continuidad de la vida laboral. Así, en contraste con el modelo de biografía laboral prolongada y lineal propia del tiempo fordista masculino, nos encontramos hoy con un modelo de biografía laboral severamente adelgazado y discontinuo. Las expectativas laborales, el segundo aspecto temporal que nos interesa, se encuentran estrechamente ligadas a la definición o forma de las biografías laborales. Las expectativas, por dar una breve definición, conforman los horizontes de futuro sobre los que los trabajadores proyectan sus vidas, guiando las acciones y las decisiones de su presente. Castro (2008) sostiene que, con la diversificación de los tiempos de trabajo, no solo se ha dado una ampliación del campo u horizonte de las expectativas laborales, sino que ha crecido inusitadamente la impredecibilidad ligada a los distintos aspectos temporales del trabajo: la duración de los contratos, de las jornadas y de los calendarios laborales, la regularidad de los horarios y el orden o la secuencia de la trayectoria profesional. El espacio de las expectativas se diversifica, se complejiza y se llena de incertidumbres. En este sentido, podemos decir que ambos aspectos, diversificación de las biografías y expectativas laborales, reconocibles en las experiencias habituales de las mujeres en el mercado laboral, contribuyen a la idea de un "tiempo de trabajo femenino" que crece frente a uno masculino-industrial.

Yendo un paso más allá del excelente análisis que propone de Castro, en el presente artículo defendemos que, tanto la redefinición de los ciclos laborales, como la de las expectativas laborales, no solo afecta 
al espacio de proyección laboral, sino que también está ocasionando un importante impacto sobre la planificación vital. Ciertamente, la centralidad que adquiere el trabajo en el ordenamiento de la vida en la época industrial y el peso que mantiene aún hoy en los imaginarios sociales (Prieto, 2007), provoca que las complicaciones temporales que nacen en el ámbito del trabajo, se transfieran al ámbito de la vida. Si defendemos que las expectativas biográficas de los individuos determinan tanto sus acciones como su toma de decisiones, podemos deducir que las transformaciones de los tiempos de trabajo, por el peso determinante que muestra este sobre los aspectos vitales, están desgastando los contrafuertes sobre los que se apoyaban estas expectativas, erosionando del mismo modo su capacidad de acción y de toma de decisiones en el terreno de la vida. Sintéticamente: la diversificación de las biografías y de las expectativas laborales complica el manejo de las expectativas vitales, traspasando la incertidumbre que se gesta en lo laboral al entorno de las acciones y decisiones de la vida.

\section{LA DESESTANDARIZACIÓN DEL CICLO VITAL Y LA INDIVIDUALIZACIÓN DE LAS BIOGRAFÍAS}

En los últimos años, en estrecha relación con lo expuesto, aunque especialmente centrado en los estudios del ciclo vital, de la juventud y de la transición a la edad adulta, ha surgido un intenso debate en torno a la tesis de la desestandarización del ciclo vital. En este debate, observamos dos posturas claramente contrapuestas: quienes abogan por la teoría de la desestandarización del ciclo vital y quienes defienden la persistencia de la forma estandarizada de este. Las partes que abogan por la persistencia del ciclo vital estandarizado (Elchardus y Smitts, 2006; Fenton y Dermott, 2006) toman la base de su argumento en el tiempo fordista o de la norma social del empleo (Alonso, 1999; Alonso, 2007; Prieto, 2007). Como apuntábamos unas líneas más arriba, las protecciones ligadas a los estados de bienestar y la fuerza del modelo de producción fordista posicionaron el trabajo masculino como uno de los elementos de ordenación centrales de la época dorada del capitalismo (Prieto, 2007). Las lógicas normativas de la época, como la legislación laboral o educativa (edad de jubilación, edad de escolarización obligatoria...), con el trabajo como centro, estructuraron el tiempo de vida o tiempo biográfico, principalmente masculino, a través de la secuencia vital "formación-trabajo-retiro" (Alonso, 2007) o, como denominan Kohli y Rein (1991), mediante "la triangulación del ciclo vital".
Se da una fuerte homogeneización del ciclo vital, abonando el terreno para la extensión de un modelo biográfico altamente estandarizado y caracterizado por un orden secuencial que divide la vida en etapas y transiciones claramente pautadas y diferenciadas. Este orden queda anclado en las concepciones generales del tiempo, afianzándose profundamente en los imaginarios temporales y culturales de las gentes de la época (Leccardi, 2005) y marcando tanto su modelo biográfico de referencia como sus expectativas de vida: un trabajo lineal, estable y protegido que posibilita desarrollar la vida dentro de un orden lineal, estable y predecible. Es el tiempo de las biografías ordenadas y las expectativas fiables.

Si bien es cierto que los defensores de la tesis de la desestandarización (Beck, 1992; du Bois-Reymond y López Blasco, 2004; Bradley y Devadason, 2008; Furlong y Cartmel, 1997; Stauber y Walther, 2006) comparten este primer momento de diagnóstico de los continuistas defensores de la estandarización, aducen que las estructuras que soportaban la secuencia vital estandarizada o las "biografías normales" entran en declive con la crisis de los años 70 o, lo que es lo mismo, cuando los dos elementos principales que las sostenían, los estados de bienestar y el modelo de producción fordista, comienzan a desgastarse. Según estos autores, los principales contrafuertes que soportaban la secuencia "formación-trabajo-retiro" (y todo lo que ella implicaba) se ven en apuros. Luís Enrique Alonso, en intensa relación con esta idea, expone que las fases suaves de lo laboral, es decir, estas que posibilitaban la entrada de los jóvenes de forma progresiva al mercado laboral (primera transición: formación-trabajo) y preparaban a los más mayores para su abandono (segunda transición: trabajo-retiro), y que permitían sostener un ciclo vital basado en tres etapas (formación, trabajo y retiro), empiezan a mostrar un importante deterioro. Este hecho, en palabras del autor, da lugar a un estrechamiento paulatino de la zona laboral central y repercute en las pirámides de edad del mercado laboral, generalizando un ciclo biográfico que concentra su etapa "más laboral" en el centro de la vida, mientras precariza o inestabiliza sus extremos (Alonso, 2007) o las experiencias laborales de aquellos colectivos más vulnerables.

Añadiendo más elementos a este diagnóstico, los autores que abogan por la tesis de la desestandarización del ciclo vital afirman que, en este tiempo, el ciclo vital, además de verse desprovisto de sus pautas compartidas, comienza a ser gobernado por decisiones individuales, relacionando la tesis de la desestandarización 
con la tesis de la individualización ${ }^{3}$. Desde este esquema, y como fruto principal de las decisiones biográficas individuales, estos autores plantean el paso de unas biografías estandarizadas a unas biografías electivas o "choice biographies" (Beck, 1992; du Bois-Reymond, 1998; Furlong y Cartmel, 1997; Wilkinson, 1997). La individualización en la toma de decisiones, señalan, deriva en una diversificación de las posibles rutas de avance vital donde los tiempos varían, las secuencias se flexibilizan, los límites de las transiciones se diluyen, las etapas se interrumpen o se retrasan... Leccardi (2005), compilando lo dicho, habla del fin de la secuencia tradicional ordenada. Con todo esto, los representantes teóricos de la desestandarización defienden que el ciclo de vida entendido como una secuencia estandarizada de etapas y transiciones es sustituido por un ciclo vital expuesto a una multiplicidad de opciones y posiciones dentro de las cuales los individuos deben conformar su trayectoria vital determinados por unas importantes dosis de incertidumbre.

Coincidiendo con los argumentos teóricos de los defensores de la desestandarización, en este artículo defendemos que, del mismo modo que los ciclos divididos en tres etapas basados en la centralidad y las características propias del trabajo masculino de la época dorada del capitalismo constituyeron un elemento de ordenación que ayudó a construir y consolidar un modelo biográfico pautado o una biografía estandarizada, el desgaste o la expansión de la precarización de sus estructuras y su pérdida de centralidad han provocado un proceso de desestandarización o diversificación de las posibilidades biográficas. Atendiendo a los resultados empíricos de ambos posicionamientos, no obstante, encontramos una contradicción sobre la que es necesario detenerse y reflexionar. Por una parte, los resultados de las investigaciones de los que abogan por la persistencia del ciclo vital estandarizado nos señalan que el ideal estandarizado perdura y predomina en los imaginarios de los individuos encuestados. Por otra parte, las conclusiones de los partidarios de la desestandarización apuntan en la dirección opuesta, al mostrar prácticas y estrategias que describen una alta diversidad en las rutas o trayectorias vitales de los individuos entrevistados.

En nuestro caso, y atendiendo a cuestiones trabajadas con anterioridad (Artegui Alcaide, 2014), podemos afirmar que, ciertamente, en el caso de los jóvenes del perfil recogido, el ideal laboral que predomina es el sujeto a la norma social del empleo masculino, es decir, un ideal de trabajo lineal, estable y ascendente. Avanzando un paso más, no obstante, nos parece importante hacer mención de aquella contradicción que nos ayuda a posicionarnos del lado de la desestandarización dentro de este debate, y es que este ideal laboral, en la mayoría de los casos estudiados, solo funciona en el nivel simbólico. Dicho de otra manera, a pesar del peso que muestra este ideal en los discursos producidos por los jóvenes, la mayor parte de sus experiencias laborales se asemejan mucho más a la norma femenina a la que venimos haciendo referencia a lo largo del artículo. Esta contradicción entre lo simbólico y lo vivencial parece responder al carácter transitorio que achacan los jóvenes a su situación laboral. No obstante, la permanencia en el tiempo de estas situaciones desfavorables, como veremos en las conclusiones, está comenzando a poner en duda tanto la hipótesis de la transitoriedad como la propia confianza en el modelo.

Con todo lo recogido y siempre con afán de contribuir a resolver el debate en torno a la desestandarización, exponemos que el elemento que parece estar determinando unos y otros resultados no es solo el paradigma interpretativo desde el que los investigadores se aproximan a esta realidad, sino las limitaciones de la metodología que utilizan para acercarse a ella. Así, entendemos que un estudio de corte cuantitativo (como es el caso de los defensores de la persistencia) es válido y relevante para comprobar el nivel de asentamiento simbólico del modelo biográfico estandarizado en la actualidad, pero que corre el peligro de dejar fuera matices importantes ligados a las prácticas, las experiencias y las contradicciones de la propia experiencia juvenil. Un estudio de corte cualitativo (como el que utilizan los representantes de la desestandarización), por contra, muestra mayor capacidad para atender tanto a lo simbólico, como a las novedades y contradicciones que se están generando en la práctica transicional, a pesar del fuerte asentamiento del ideal estandarizado fordista. Con todo, en este estudio defendemos que, con mayor o menor peso de cada una de las versiones, tanto el ciclo vital estandarizado como sus versiones desestandarizadas están conviviendo en la actualidad. Y es esta coexistencia, precisamente, la que nos lleva a analizar este terreno desde una mirada ecuménica que trata de no privilegiar una visión frente a la otra, sino de dar parte de su totalidad.

\section{EL IMPACTO DE LA INCERTIDUMBRE EN LA TRAN- SICIÓN Y SUS EFECTOS}

A pesar de partir de una postura global, en este artículo queremos mostrar cómo el impacto de la incer- 
tidumbre sobre los jóvenes que siguen guiando sus vidas por la secuencia estandarizada no es el mismo que sobre aquellos que la han abandonado. De la misma manera, esta distinción nos permitirá exponer que los efectos o las consecuencias de esta sobre las vidas serán diferentes en unos perfiles y en otros.

Para comprobar estas aseveraciones, analizaremos de qué forma impacta la incertidumbre en las vidas de aquellos jóvenes más próximos al ideal estandarizado y de qué manera lo hace en aquellos más cercanos a la desestandarización. Con el fin de recoger las características específicas y determinantes de los jóvenes adscritos a cada grupo, atenderemos a cuatro elementos concretos: sus especialidades formativas, las principales características asociadas a sus trayectorias laborales, si han sufrido un choque de expectativas y cómo lo han afrontado o interpretado en caso de sufrirlo y el nivel de distanciamiento de sus bases de socialización ${ }^{4}$. En lo que se refiere a sus especialidades formativas, predecimos que tanto la inserción laboral como las condiciones de trabajo no serán iguales en los perfiles técnicos que en el resto de perfiles formativos. En lo que se refiere a las características asociadas a sus trayectorias laborales, intuimos que el impacto de la incertidumbre no será el mismo en aquellos jóvenes que muestren unas trayectorias lineales y estables que en aquellos que muestran niveles notables de inestabilidad, niveles fuertes de precariedad o una intensa flexibilidad. En lo que se refiere al choque de expectativas, creemos que el impacto de la incertidumbre será distinto entre aquellos jóvenes que han visto frustradas las expectativas que construyeron en el pasado que entre aquellos que las han podido cumplir sin mayor problema. Finalmente, en el caso del distanciamiento de las bases de socialización, suponemos que el grado de arraigo o desarraigo que muestren los jóvenes frente a las pautas o parámetros de avance vital recibidas a lo largo de su vida dará lugar a diferentes consecuencias asociadas a la incertidumbre.

Con todo lo dicho y atendiendo a los datos producidos mediante el trabajo de campo, podemos observar que cada una de las posturas que hay que analizar guarda en sí una amplia diversidad de matices que genera nuevas distinciones o subgrupos de análisis. En el caso de la persistencia del ciclo vital, son dos los subgrupos que detectamos: los estables y los inestables. En el caso de la desestandarización del ciclo vital, encontramos también dos subgrupos: los precarios y los flexibles.

\subsection{La persistencia del ciclo vital: estables e inestables}

En lo que se refiere a los jóvenes más cercanos a la persistencia del ciclo vital, el primer subgrupo que encontramos es el conformado por los estables. Se trata de jóvenes con trayectorias formativas técnicas; en su mayoría, ingenieros. Este tipo de perfiles profesionales son los que mayor tasa de inserción laboral y mejores condiciones de trabajo están mostrando en la actualidad. En concordancia con esto, los jóvenes insertos en este subgrupo afirman vivir unas trayectorias laborales sin sobresaltos. El paso de lo formativo a lo laboral fue fluido $y$, si bien es cierto que en el comienzo de sus trayectorias laborales las condiciones fueron algo peores de lo que esperaban, todos coinciden en afirmar que estas, en la actualidad, y en términos generales, cumplen con lo esperado:

\footnotetext{
"Yo conseguí una beca para hacer el proyecto de fin de carrera y luego tenías opción a quedarte allí. (...). Empecé de becaria. Luego me pasaron por ETT, me subcontrataron a través de una ETT. Y eso fueron dos años. La beca un año. Cobrábamos unos setecientos y pico o ochocientos euros al mes, eran siete horas y media, creo... que eran al día siete horas. Al principio empezamos a media jornada, o sea cuatro horas y media o así, y cobrábamos igual unos quinientos, cerca de quinientos euros. Yo a media estuve poquito; estuve tres meses o así y luego el resto del año estuve a jornada completa. (...) En principio ellos decían que todo estaba sujeto a cómo ibas trabajando tú, pero en principio sí que... (...) quiero decir, toda la gente que conozco ha entrado" (Ingeniera, 31 años).
}

"Lo mío era una decisión muy fácil: «voy a hacer esta carrera porque sé que es buena, y lo sé tanto, que no tengo ni puta idea y ni siquiera necesito cuestionármelo. Como todo el mundo sabe y en el aire está escrito que esta carrera es buena, ni siquiera tengo que pensar sobre ello: la cojo y ya está» (...). Mis expectativas laborales han encajado con lo que yo pensaba. Yo tengo unas condiciones súper buenas. Igual pensaba que iba a tener más dinero... siempre he pensado que por ser ingeniero iba a tener bastante dinero" (Ingeniero, 32 años).

Basándonos en los testimonios producidos a través del trabajo de campo, observamos que, en el caso de estos jóvenes, no se da un choque entre las expectativas que construyeron en el pasado y sus vivencias del presente. Sus vidas, tanto en lo laboral, como en lo vital, transcurren como las habían programado. Esta es la principal razón por la que estos jóvenes, por lo general, no muestran un fuerte distanciamiento hacia sus bases 
de socialización. Es decir, el hecho de que sus proyectos se hayan desarrollado con arreglo a sus expectativas, les lleva a no poner en cuestión la validez de las pautas o guías que recibieron en su proceso de socialización y su aplicabilidad en la actualidad. La persistencia de la secuencia estandarizada, en este caso, responde a la validez que ha mostrado esta en unas condiciones laborales como las descritas en este grupo.

Si analizamos el impacto de la incertidumbre sobre la vida de estos jóvenes, podemos observar que, si bien es cierto que son conscientes de que vivimos un periodo en el que las certezas son difíciles de encontrar, la estabilidad y la predictibilidad que se deriva de sus trayectorias laborales hace que su impacto no se sienta en primera persona.

"Todo ha venido bastante rodado. Nunca he tenido que sacrificar nada de nada por el trabajo (...). Mi camino es recto y vivo en él (...). Es que una linealidad muy lineal, o sea, es una recta, recta-recta... (...) es como cuando vas por una carretera de California: solo ves un punto de fuga" (Ingeniero, 32 años).

“Hombre, yo me siento un privilegiado. (...) A mí siempre me han planteado «tú te quedas para toda la vida; es lo que queremos» (...). Y la verdad es que me siento un privilegiado: las cosas como son. (...) Yo me veo con estabilidad. (...) No lo veo complicado porque tanto laboralmente como económicamente estoy bastante desahogado. No veo que mi trabajo peligre y creo que económicamente estoy por encima de la media, con lo cual, eso no creo que sea un problema en mi caso. Pero eso en mi caso, que me siento un privilegiado. Todos los días me repito: "Virgencita, que me quede como estoy»" (Ingeniero, 30 años).

El segundo subgrupo de esta categoría, los inestables, está conformado por jóvenes con trayectorias formativas diversas, que van desde arquitectos, licenciados en Biología, Farmacia, Administración y Dirección de Empresas y Bellas Artes hasta ingenieros de la rama de la construcción (dentro de las ingenierías, es una rama fuertemente dañada por la crisis inmobiliaria) o diplomados en Magisterio. Si atendemos a la extracción de datos derivada de las entrevistas, podemos afirmar que, a diferencia de las del primer subgrupo, las trayectorias laborales de estos jóvenes muestran notables signos de inestabilidad. Esta, por lo general, se encuentra asociada a la alta tasa de rotabilidad a la que se han visto sometidos a lo largo de sus cortas trayectorias de trabajo. Rotabilidad que, por otra parte, no es fruto de la decisión o ambición propia, sino de la propia dinámica del mercado de trabajo:
"Estuve en una guardería privada, luego pasé a las Haurreskolak [consorcio de escuelas infantiles] y luego al Gobierno Vasco. Luego, en el Gobierno Vasco, como va por sustituciones, pues te van llamando. En el Gobierno Vasco llevo como seis años. Habré pasado como por treinta colegios. Son pocos (se ríe). (...). Ahora aspiro a una plaza. Yo creo que me va a costar más, pero lo voy a conseguir".

“- ¿Qué supone tener una plaza?

- ¡Un año de estabilidad! (se ríe). Una estabilidad. Un año que puntúas seis puntos enteros, con lo cual el año que viene también vas a poder coger otra plaza porque tienes más puntos. Sabes que vas a cobrar navidades, semana santa, verano..." (Diplomada en Magisterio, 30 años).

“¿Estabilidad? Sí... ya... jel 31 de diciembre se me acaba el contrato! Cuando me hicieron las entrevistas, pero claro, esto fue hace dos años, no existía la crisis que hay ahora, ni existían los recortes que hay ahora actualmente (...). No estoy muy segura de que esto vaya a tener continuidad (...). Nunca me he sentido estable, porque nunca me han dado la posibilidad de estar estable" (Licenciada en Biología, 29 años).

Cuando preguntamos a los jóvenes sobre las expectativas que construyeron en el pasado y sus vivencias del presente, todos coinciden en afirmar que se ha dado un choque entre lo primero y lo segundo. En ninguno de los casos sus experiencias del presente casan con los planes por los que se esforzaron años atrás. La fractura se relaciona con la situación laboral que viven:

“Tampoco pensaba montarme en el dólar pero tampoco pensaba que me iba a costar tanto trabajo encontrar trabajo. O sea, cuando empiezas la carrera y en esa época, en menos de un mes y medio estabas colocado en una empresa, ya fuese de construcción, ya fuese de energía, ya fuese... entonces, después del esfuerzo, salir y no encontrar trabajo en seis meses pues... de ahí el bajón" (Ingeniero, 30 años).

"Yo esperaba tener más estabilidad laboral y tener un contrato... un tipo de trabajo quizás con más responsabilidad, porque, al final, tengo veintiocho años, ¿sabes? Y no puedo estar toda la vida de becaria. Sí que esperaba tener más responsabilidad, un puesto no tan junior, un poquito más arriba... pero no ha podido ser" (Licenciada en Administración y Dirección de Empresas, 28 años).

A pesar de encontrarse con este conflicto de expectativas, observamos que el nivel de distanciamiento que 
muestran estos jóvenes hacia sus pautas de socialización es escaso o nulo. La principal razón asociada a este fenómeno es la explicación que encuentran estos jóvenes al choque de expectativas: la situación de contrariedad que viven no es más que una consecuencia de la actual coyuntura (crisis, desempleo juvenil...).

"Esta crisis va a acabar. Yo me considero positiva en ese aspecto. Estoy hasta las pelotas de la gente que se piensa... ¿qué pasa, que nos vamos a quedar todos en el paro?, ¿toda España se va a quedar en el paro? No, joder. Esto pasa. Esto es una bajada y una subida. Si a mí me estás diciendo que cada año va a ser peor, ¿por qué?, ¿porque lo dices tú?, ¿porque has leído un par de cosas o porque has visto la tele? Que sí, que estarán jodidas las cosas, no sabemos lo que va a durar, pero en algún momento esto desaparecerá (...); entonces, cuando la cosa remonte, la gente volverá a lo mismo" (Licenciada en Bellas Artes, 29 años).

"Yo creo que hay que aguantar. Aguantar un poco hasta que pase esto. Un par de años... un año... y luego ya saldrán las cosas. Yo creo que con el tiempo ya saldrán las cosas, ya se irán... sí... no sé... A ver, yo tengo la sensación de que igual la movida está muy chunga y tal, pero yo tengo el deseo o la esperanza de que no vaya a ser tan heavy; de que sí que pasemos un par de años putos y luego ya empezar a salir" (Diplomada en Magisterio, 30 años).

Con esto, como señalábamos al inicio, los jóvenes entienden que la que viven es una situación transitoria, que pasará con el tiempo y que todo volverá a su cauce normal. Así, en este caso, podemos decir que la persistencia de la secuencia estandarizada y de las pautas ligadas a ella es fruto de una lectura optimista de las expectativas de vida que construyeron y la situación que viven a día de hoy.

Esta lectura, no obstante, está teniendo sus efectos sobre los transcursos vitales de estos jóvenes. El hecho de no poder avanzar en sus planes según lo habían programado, está provocando que sus vidas se guíen por dos estrategias que les encierran en el presente: la espera y la adaptación. Viendo que no pueden avanzar según las premisas que esperaban, estos jóvenes están dejando sus planes congelados hasta que puedan volver a desarrollarse y cumplirse en las condiciones que les prometieron. En algunos casos, y por la propia frustración que les genera este estancamiento, hacen pequeñas adaptaciones de sus planes con el fin de avanzar poco a poco.

“Creía que todo iba a ser mucho más... conseguir esas cosas... tenía en mente que iba a ser antes. Cuan- do estaba en el instituto, me veía que con veintiséis años, más o menos, ya iba a tener la vida un poco... iba a tener mi trabajo, mi casa... yo que sé... imi familia empezada por lo menos! Y ahora veo que eso se ha ido, tu tu tu (onomatopeya), atrasando, ¿no? Mi hermana con veintisiete años tuvo a su hija; yo tengo 30 y todavía... ivete a saber! Quiero decir (...) tienes que amoldarte" (Diplomada en Magisterio Infantil, 30 años).

"Pues me ha afectado bastante. A mí me hubiera gustado ser padre antes de los veintinueve. No creo que sea especialmente mayor, tampoco creo que sea joven (...) pero ser padre con veinticinco o veintiséis sí me hubiera gustado. Ahora estoy encantado, pero ser padre con veinticinco o veintiséis hubiera sido una gozada. Y lo tuve que retrasar por todo esto [inestabilidad laboral]" (Licenciado en Biología, 29 años).

"Acabas adaptando tus planes. Quiero decir... pues si igual has soñado con tener tres hijos, pues igual te quedas con uno, pero has tenido tus hijos. $O$ igual en vez de tener una boda de trescientos invitados, tengo una de cincuenta, pero es tu boda. O sea, al final la gente se adapta y sigue. Pero sigue. Sí sigue con su vida. Yo creo que la gente no está aparcándolo tanto... Igual sí que lo retrasa un par de años como mucho, pero no indefinidamente: al final lo que tienes en mente al final lo acabas haciendo o buscas la manera de hacerlo" (Licenciada en Biología, 29 años).

A través de los testimonios de los jóvenes de este grupo, se puede afirmar que, a pesar de que la secuencia estandarizada sigue presente en sus imaginarios, la incompatibilidad que muestra con sus vivencias y experiencias hace que se convierta en uno de los principales problemas asociados a su dificultad para avanzar.

En lo que se refiere al impacto de la incertidumbre, podemos decir que, si bien es una cuestión que aparece de forma puntual en sus discursos, su protagonismo es menor y se manifiesta de forma abstracta o poco concisa, haciendo referencia a situaciones posibles o probables y siempre relacionadas con el ámbito laboral, y no tanto vital:

"Al final es un poco difícil... incertidumbre total, sí. Tienes que vivir pensando si dentro de seis meses vas a tener que ponerte un traje o a la de doce meses te vas a tener que poner un mono para barrer. Pues un poco de incertidumbre... Es difícil, pero bueno, de momento, ¿no? Tiramos para adelante" (Ingeniero, 30 años).

"Yo creo que el propio tiempo, o sea, tú puedes estar preocupado un día por algo, dos días, tres días, y 
al final parece como que lo vas mitigando; y se queda ahí. A veces parece que rebrota y piensas: «que se me acaba, que se me acaba» o «me voy a quedar en la calle», pero bueno, no es porque hayan mejorado las cosas, todo lo contrario, no han mejorado..." (Licenciado en Biología, 29 años).

El principal razonamiento que encontramos a esta situación está relacionado con los "presentes congelados" a los que hacíamos referencia anteriormente. Es decir, el hecho de que estos jóvenes se hayan quedado atrapados en el presente y a la espera de buenas señales que les permitan avanzar hacia el futuro proyectado hace que la sensación de incertidumbre ligada al futuro se bloquee. Así, el hecho de no dar pasos arriesgados, o no adentrarse en zonas oscuras o que se salen de la secuencia estandarizada, provoca que la sensación de incertidumbre pierda protagonismo.

\subsection{La desestandarización del ciclo vital: precarios y flexibles}

En segundo lugar, en lo que se refiere a los jóvenes más cercanos a la desestandarización del ciclo vital, observamos también la preeminencia de dos subgrupos. El primer subgrupo, los precarios, está conformado nuevamente por una amplia variedad de perfiles formativos: licenciados en Psicología, Bellas Artes, Historia del Arte o Periodismo y, eventualmente, algún arquitecto. La generalidad de estos perfiles formativos (exceptuando los arquitectos), como hemos podido observar en las entrevistas, son considerados como perfiles con una menor tasa de inserción laboral y de menor éxito en el mercado. Se trata de profesiones que responden en mayor medida a deseos de autorrealización o vocación personal que a la demanda del mercado.

“Estudié Bellas Artes. Había varias opciones y supongo que esa es la que me pareció más idónea... más idónea no, más apetecible, no idónea (...) básicamente no estaba pensando en mi futuro, o sea, es así de sencillo. Si volviera a empezar no haría lo mismo" (Licenciado en Bellas Artes, 31 años).

"Había como una norma social con las carreras, sobre todo con la mentalidad de mis padres que son personas que son de otra generación (...) eso se ha visto luego, el gran engaño de «si vas a la universidad vas a estar bien preparado luego" y ahora se han visto que las cosas... las ingenierías y empresariales igual sí, pero las humanidades y la cultura... no hay curro" (Licenciada en Periodismo e Historia del Arte, 32 años).

En el caso de los jóvenes de este subgrupo, atendiendo a sus trayectorias laborales, podemos obser- var que, por lo general, la inestabilidad y la precariedad son los dos elementos que sobresalen. A pesar de mostrar una fuerte rotación, como en el caso de los jóvenes representados en el segundo subgrupo presentado (inestables), debemos señalar que los periodos de desempleo o las malas condiciones laborales son notablemente superiores en estos:

“¿Quieres número de trabajos o clases de trabajos? Número... infinito, o sea, incontable. $O$ sea, en audiovisuales tienes muchos trabajos de un solo día, de dos días... con suerte coges una peli... Paralelamente haces otras cosas por otro lado. No sé... simplemente es incontable el número de veces que puedes llegar a trabajar en un año, porque ves, por ejemplo, el número de pagadores que has tenido... ihojas! O sea... Pero he tenido trabajo que me ha dado dinero por otra parte $y$ he tenido alguna época de tener que hacer algún curro completamente ajeno a digamos la formación que he tenido" (Licenciado en Bellas Artes, 31 años).

"Yo tenía unos ahorros y fui tirando con ellos. Aparte, estoy dando clases a chavales de bachillerato y de la ESO (...). Y desde después de verano también me están ayudando mis padres a pagar el alquiler y tal. No, ahora mismo del estudio, aparte de que nos deben pasta y tal, pero no nos da para vivir al cien por cien" (Arquitecto, 31 años).

"Yo siempre he sido independiente, pagando poco alquiler o pagando con retrasos (...), que sí ha habido épocas que no he podido, luego se lo he dado o lo que sea (...). Yo ahora estoy cobrando la ayuda [Renta de Garantía de Ingresos]. Desde febrero" (Licenciada en Periodismo e Historia del Arte, 32 años).

El choque de expectativas es reconocido y abalado por todos los miembros de este subgrupo. En este sentido, y en contraste con el subgrupo analizado anteriormente (inestables), observamos que este choque es el principal elemento que les lleva a distanciarse de las pautas de socialización en las que fueron educados. Esto, por lo general, surge de la sensación de engaño que impera en dichos jóvenes.

"Y te encuentras pues con todo lo contrario. La burbuja ha estallado, ¿no? Lo laboral ahora es inestabilidad, incertidumbre y mucha ansiedad también (...). Lo que nos prometieron era eso, era: «si haces esto, vas a tener esto" y ahora eso no se corresponde, ¿no? Entonces estamos también un poco perdidos, porque dices: «joé, si he hecho todo lo que me han pedido, ahora ¿por qué no estoy recibiendo todo lo que me prometieron?»" (Arquitecto, 31 años). 
La sensación de incertidumbre en el caso de estos jóvenes es intensa. Por una parte, observamos que se alejan de los parámetros de vida con los que crecieron en sus etapas tempranas. Por otra parte, no obstante, no se sienten dueños de sus propias vidas. Afirman que sus presentes y sus futuros están determinados por las acciones y las decisiones de agentes externos a ellos y que, frente a sus caprichos, poco o nada pueden hacer. Esto les genera un fuerte sentimiento de frustración, al dejar en evidencia que no son ellos quienes guían sus vidas o, como señalan ellos mismos, al dejar patente que no tienen el control sobre sus vidas o sus futuros.

"Son dientes de sierra, como nos enseñan en historia, no sé lo que va a durar el piquito de abajo, pero espero que no sea toda mi vida, porque me joden vivo, a mí y a cualquiera (...). Y, sobre todo, que está montado de tal manera que yo no puedo hacer nada, ni yo, ni tú, ni nadie. (...) Yo no tengo control, sobre todo a día de hoy, no tengo herramientas para decir: "voy a redirigir mi situación»" (Licenciado en Bellas Artes, 31 años).

"Pues un poco de impotencia, por una parte, porque creo que no tengo ninguna capacidad de decisión; al final lo que siento es que la decisión no está en mí, sino que está en una parte fuera y simplemente tengo que lidiar de la mejor forma que pueda con ella y eso me genera una frustración horrible: al final no soy actor, soy como tele-observadora en el teatro de mi vida, en el que cada vez va peor y en el que yo no puedo hacer nada" (Licenciada en Psicología, 30 años).

A pesar de lo dicho, se muestran contradictorios en la lectura de su situación personal: aunque opinan que su devenir no está en sus manos, la imposibilidad de interpretar su realidad con claridad les lleva a erigirse como los principales culpables de sus situaciones actuales. Así, lo que en un principio se expone como una sensación de frustración o descontrol, acaba presentándose como un fracaso personal por no haber sabido elegir bien, por no haber estado más despiertos, por no haber sido capaces de hacerse cargo de sus vidas.

"Es como: «bueno, he fracasado. Literalmente, he hecho una apuesta y la he cagado. No sé cómo, ni porqué, pero hemos llegado hasta aquí». (...) Es un castillo de naipes que se ha venido abajo a las primeras de cambio, si lo quieres ver así. (...) Te pongas como te pongas, siempre vas a tener la idea de que has hecho algo mal. (...) Estoy frustrado, o sea, solo quiero sobrevivir. Ya me he olvidado de... no tengo un modo de vida idílico, simplemente es sobrevivir (...) ya no tengo una idea de a dónde tengo que llegar" (Licenciado en Bellas Artes, 31 años).

"Es mi culpa. No me lo he montado bien, porque podía haber hecho más, o menos (...) si hubiera tomado otras decisiones igual hubiera ido la cosa mejor, pero bueno, habría sido todo distinto (...), pero igual no sería feliz... tampoco soy feliz ahora..." (Licenciada en Periodismo e Historia del Arte, 32 años).

Con esto, podemos afirmar que, en el caso de los jóvenes ligados a este subgrupo, la sensación de incertidumbre es fuerte y las consecuencias que vierte sobre sus vidas graves. La situación de contrariedad que viven les está llevando a una fuerte corrosión de su carácter y al establecimiento de la inseguridad y el descontrol como elementos perpetuos en sus vidas.

El segundo subgrupo de esta categoría, aquellos jóvenes que caracterizamos como los flexibles, también responde a una amplia variedad de perfiles formativos: licenciados en Derecho, Ciencias Políticas, Sociología, Humanidades y Empresa, Administración y Dirección de Empresas, diplomados en Comercio Internacional o Magisterio y arquitectos. Atendiendo a las principales características que describen las trayectorias laborales de estos jóvenes, podemos decir que el elemento más llamativo es su flexibilidad y movilidad laboral. Si bien es cierto que la rotación laboral en el caso de estos jóvenes es un elemento destacable y característico, hemos de puntualizar que, a diferencia de los anteriores perfiles, la rotación es fruto de sus propias decisiones, aspiraciones o deseos. Por lo general, se trata de jóvenes que, dentro de sus trayectorias profesionales, se han movido por distintas empresas y distintos países y que muestran un firme alegato a favor de la flexibilidad, cualidad que les permite crecer laboralmente, pero sobre todo personalmente. La esfera laboral se fusiona con la vital, buscando en el trabajo elementos como la autorrealización, la motivación, la vocación, el crecimiento personal, la creatividad...

"Yo podía haber opositado para sacarme un puesto en la administración bien remunerado y con muchísimas vacaciones y menos horas de trabajo, pero no he querido (...) la estabilidad que se ofrecía por ahí, de acabar la carrera y opositar, a mí me parecía el horror: primero, yo no quiero opositar; segundo, no quiero ser funcionaria por el hecho de ser funcionaria: yo quiero un trabajo en el que esté yo motivada y que pueda aportar y que tenga mi... que pueda desarrollar mi creatividad y que le pueda dar al coco" (Licenciada en Derecho, 34 años). 


\begin{abstract}
“Me gustaría tener un trabajo que me dejase tiempo para mí, para la gente con la que quiero compartir mi vida, para desarrollarme como persona también fuera del trabajo. Pero también me gustaría tener un trabajo que me desarrolle como persona (...) que me suscite curiosidad, que me haga aprender, que me genere inquietudes... un trabajo que me dé para vivir, no mucho más, no necesito mucho más, que me dé para vivir de forma sencilla (...) que me dé flexibilidad en horarios de trabajo o en días de trabajo" (Licenciado en Derecho y Ciencias Políticas, 35 años).
\end{abstract}

En el caso de estos jóvenes, detectamos un choque de expectativas que no se deriva de la imposibilidad de reproducir la secuencia en la que han sido educados, sino del hecho de no querer reproducirla. El modelo biográfico secuencial recibido no encaja con sus planes de vida flexibles. Además, se muestran críticos hacia su posible validez en las actuales condiciones socio-económicas o laborales.

"Sí que tengo la sensación de que la gente que intenta escapar de ahí es porque no le gusta ese modelo. Y porque necesita un modelo alternativo o porque no quiere ningún modelo. (...) Al final, hay peña, y yo creo que somos mucha peña, que no le gusta estar tan sumamente encorsetado en esos parámetros y entonces intentas hacerlo de otra manera, más o menos alternativa o más o menos transgresora" (Diplomado en Magisterio, 33 años).

"No me gusta ese modelo primeramente porque no es nada flexible, depende de muy pocos parámetros. Entonces, cualquier sistema que dependa de pocos parámetros... el porcentaje de catástrofe en cuanto falle alguno de ellos es mucho mayor. Entonces, es un sistema totalmente obsoleto para mí, que no me aporta nada; es contrario a lo que promete: promete seguridad, pero no es nada seguro, porque a nada que falla algo se viene abajo" (Arquitecto, 33 años).

La postura crítica que muestran estos jóvenes hacia la secuencia estandarizada del ciclo vital y el deseo manifiesto de querer ser dueños de sus decisiones y pilotos de sus vidas reafirma la hipótesis de los modelos biográficos desestandarizados e individualizados que se alejan de la linealidad secuencial.

"Yo he dejado de pensar que mi trayectoria es lineal porque... puf... porque, tío, las personas no somos lineales (...) lo que te pasa es como orgánico más que una puta línea recta y creo que la idea de plantear tu vida como una línea recta... no creo en esa idea ya... antes igual sí que decía: «hay que planear aquí el tema», pero ¡buf! Ahora creo que es mejor ser flexible" (Licenciada en Administración y Dirección de Empresas, 28 años).

"El otro modelo es más pasivo, tiene menos control de cada uno y es totalmente dirigido. Es una cosa que no me gusta, es una cosa que he tenido también desde crío, yo creo: nunca me ha gustado que me digan: "haz esto», "haz lo otro». La historia la escribes tú, eso lo tengo clarísimo: la historia la escribes tú. Y el camino lo haces tú" (Arquitecto, 33 años).

Los jóvenes expresan de forma continua el deseo y la necesidad de construir nuevas rutas; rutas que respondan y satisfagan sus necesidades propias y personales. Rompiendo así con el viejo ideal lineal y prefijado. Como señalan, esta ruptura con la secuencia estandarizada, hacerse con el control de sus propias vidas, genera altos niveles de satisfacción y empoderamiento.

"Yo estoy muy contenta por mí. Estoy contenta porque cuando decidí estudiar empresariales delegué mi plan de vida a lo que me decía mi padre y ahora he recuperado la responsabilidad de hacerme yo mi vida como a mí mejor me parezca (...) y sé que es muy difícil y yo me estoy intentando desprogramar de toda esa mierda (...). Para mí es un proceso de desaprender todas esas cosas que he aprendido" (Licenciada en Administración y Dirección de empresas, 28 años).

"Yo me siento más dueña de mi vida así, de la otra manera es un dejarse llevar y hacer lo que se supone que tienes que hacer. Soy más dueña de lo que hago y eso me empodera más, porque soy dueña de mis decisiones: decido cuándo sí, cuándo no, a dónde sí, a dónde no (...). Yo creo que es una madurez emocional que yo identifico en muchos de mis colegas: es adueñarte de tu vida" (Licenciada en Derecho, 34 años).

A pesar de la sensación de empoderamiento generalizada, muchos de los jóvenes hacen referencia a lo dificultoso de salirse del camino marcado. Alegan que la presión externa es muy fuerte y que genera fuertes inseguridades hacia el proceso vital que uno acomete. En ocasiones afirman que incluso han dejado de lado o han estado cerca de dejar de lado sus proyectos por estas presiones.

"He tenido que ir en contra de lo que se me estaba diciendo. O sea, he recibido mogollón de mensajes de inseguridad: «no tienes que hacerlo», «es un mal momento», "¿cómo se te ocurre? »... (...) espero no volver a caer en la misma piedra, porque al final ese es mi puto miedo y ese es el miedo que siempre me agobia, que es volver a la seguridad, que es lo que no quiero (llora). No quiero. Prefiero estar aquí, en este punto de... de... de perdida, pero me siento más 
yo, estoy más yo (llora) (...). En el fondo tú ves que la gente, mi familia... yo noto que a la vez que están de: «hostia, vaya par de cojones», también hay un: «la hostia que te vas a meter. Que se te pase pronto o iay, la hostia que te vas a meter!». Entonces es jodido, es jodido porque te sientes que vas un poco en contra de lo que hay que hacer, pero ¿qué hostias?" (Diplomada en Comercio Internacional, 35 años).

Atendiendo a la forma en la que impacta la incertidumbre en la vida de estos jóvenes, observamos que el origen de esta no se encuentra asociado tanto a factores externos (sociales, económicos, laborales...) como ocurría en otros perfiles, sino a su proceso de autoconstrucción biográfica, es decir, a la decisión de salirse del camino marcado y avanzar por sendas sin delimitar o definir. Dicho de otra manera, la incertidumbre es algo que viene dado por la sociedad contemporánea, pero también es algo que deciden introducir ellos en su vida a través de sus procesos biográficos individuales.

En cualquier caso, y ligado a ambas cuestiones, el principal elemento que asocian a la incertidumbre es la apertura del futuro. Las guías desaparecen y las opciones se multiplican. Lo destacable en este subgrupo es que, frente a los aspectos negativos asociados a la incertidumbre que hemos visto anteriormente, la tendencia es resaltar lo positivo. La sensación de pérdida es habitual, pero la agradecen: tienen opciones, no deben seguir el camino.

“Durante esos diez meses que no he tenido ni puta idea de qué pasaba, pero me empecé a sentir un poquito más libre. De hecho, era un máximo agobio, pero me sentía más libre. Era más una sensación que una realidad yo creo (se ríe) porque al final era una pérdida de cabeza de la hostia: el abanico era tan jodidamente amplio que no sabía ni por dónde coño tirar" (Diplomada en Comercio Internacional, 35 años).

"Claro que te abruma: tienes que ir poco a poco, no a lo loco. En vez de ir corriendo y encontrarte de repente con un pedazo de espacio abierto, vas poco a poco: das un paso, miras lo que hay alrededor, siguiente paso... pero bueno, eso siempre va a pasar. Tú siempre que entres a un bosque, si vas por un camino estrecho, cuando sales a una explanada del copón, al principio te abrumas: «ibuah, qué grande es esto!». Pero en el momento que sales, das un paso, si en ese momento empiezas a mirar, empiezas a controlar el espacio, das cinco pasos más y miras, al final esa explanada grande la puedes convertir en algo interesante para ti" (Arquitecto, 32años).
Coinciden en afirmar que se encuentran frente a un futuro incierto y que deben hacerse con las armas necesarias para hacer frente a las distintas incertidumbres que se les planteen en el camino. A pesar de ello, no les genera la fuerte desazón que veíamos en el anterior subgrupo. El hecho de poder emprender sus vidas desde sus deseos y necesidades hace que este escollo se asuma como un reto añadido y no lo vivan con gran preocupación. Además, el empoderamiento que genera hacerse con el timón de sus vidas, deriva en unas actitudes que les permiten hacer frente a los miedos desde lo que todos coinciden en expresar como "valentía". Se sienten preparados para hacer frente a lo que venga y se ven capaces de manejar la incertidumbre.

"Tienes la vida en el aire. Pero es que a mí esta situación no me incomoda; me mola bastante; porque es salir del puto plan. Yo me he sentido muy atrapada en el plan y he estado muchos años atrapada en el puto plan y para mí salir del plan es lo que llevo deseando desde hace muchos años. Conscientemente desde hace años e inconscientemente un buen tiempo. Yo creo que la clave es no tener miedo a los cambios y estar preparado para cualquier mierda" (Licenciada en Administración y dirección de Empresas, 28 años).

"Justo ahora estoy en ese punto: al ser un proceso tan tuyo, y el hacerte tanto caso, el miedo viene a lo bestia, pero también vienen los cojones a lo bestia (...) eres mucho más valiente. Yo ahora me siento muchísimo más valiente de lo que me he sentido nunca. Estoy dispuesta a pasarlo mal, pero por hacer lo que yo quiero; no estoy dispuesta a pasarlo mal gratuitamente, que es lo que estaba haciendo y simplemente para hacer lo que tenía que hacer. Entonces, ¿cómo lo llevo? Pues lo llevo bastante bien (se ríe). Es que al final es lo que te digo: hay mucho miedo, pero también hay mogollón de satisfacción; mogollón." (Diplomada en Comercio Internacional, 35 años).

\section{CONCLUSIONES}

Basándonos en lo expuesto, podemos afirmar que, en la actualidad, el impacto de la incertidumbre sobre las vidas no es el mismo entre los diferentes perfiles de jóvenes analizados 5 . En una primera valoración, podemos explicar que, de acuerdo con los datos producidos en las entrevistas, tanto aquellos que gozan de unas trayectorias estables como aquellos que han flexibilizado sus trayectorias se encuentran notablemente protegidos frente a los envites de la incertidumbre y sufren de forma poco dramática sus efectos. Aquellos que conviven con la inestabilidad laboral, por su 
parte, se vacunan de esta a través de estrategias de espera o adaptación, frenando, en cierto sentido, sus posibles efectos. La peor parte, en cambio, se la llevan aquellos que se encuentran en situaciones de precariedad continuada: la incertidumbre empapa sus vidas y oscurece su carácter.

Más allá de estas conclusiones, cabe explicitar que las posturas que hemos presentado no son rígidas o estancas: se trata de tipos ideales. A pesar de presentar elementos determinantes que nos permiten asociar a los jóvenes a un grupo u otro, a lo largo del análisis encontramos diferentes signos que apuntan hacia la posible tendencia a la movilidad entre perfiles. La crisis (y sus vivencias), elemento que aparece de forma transversal y no programada en el análisis, y que se hace explicita en varios de los testimonios aquí recogidos, ofrece interesantes indicios de ello. Esta, además de hacer hincapié sobre lo determinante del progresivo empeoramiento de las condiciones laborales de los jóvenes a la hora de configurar sus biografías y planificar sus futuros, parece que empieza a marcar un punto de inflexión que puede llevar a cambiar los posicionamientos de los jóvenes en el futuro, señalando ciertas pistas y cautelas que habrá que tener en cuenta en próximas investigaciones.

En esta misma línea, cabe advertir que si bien es cierto que la movilidad entre grupos no hace sino afianzar la hipótesis de la diversificación de las rutas biográficas, entendemos que es importante estar atentos a sus derivas pues, a causa de la creciente degradación de las trayectorias laborales de los jóvenes en las últimas décadas y su agravamiento tras la crisis, es muy probable que los movimientos muestren una tendencia hacia el empeoramiento respecto al impacto de la incertidumbre y sus efectos. Atender a las actitudes y estrategias que se están forjando en torno a los perfiles que presentamos como flexibles puede ayudarnos a encontrar rutas de avance más navegables ya que, frente a las otras tres posturas descritas, apuntan a ser los jóvenes que de mejor manera están entendiendo el nuevo tiempo incierto y de forma más ágil están construyendo estrategias para enfrentarlo y resolverlo.

"Yo creo que es una cuestión de mochila ligera. Si tú vas por el camino marcado, tienes menos riesgo, ya... ia no ser que lleves una mochila de cien kilos encima! Quiero decir, tienes un montón de ataduras que te entorpecen un montón de movimientos. Entonces, seguramente, llegarás allí, pero igual con unas lesiones de puta madre. Tienes que aprender a vivir con la mochila ligera" (Arquitecto, 33 años).

\section{AGRADECIMIENTOS}

El presente artículo se deriva de la tesis doctoral de la autora, inscrita en el Departamento de Sociología 2 de la Universidad del País Vasco/Euskal Herriko Unibertsitatea y financiada por el Departamento de Educación, Universidades e Investigación del Gobierno Vasco. Quisiera agradecer a Matxalen Legarreta y Ramón Ramos la riqueza que han aportado al texto a través de sus comentarios y sugerencias. Quedan, con todo, exculpados del resultado final que se presenta.

\section{NOTAS}

1. Autoras como Pilar Carrasquer y Teresa Torns (2007) han mostrado de qué manera las condiciones laborales que comienzan a dibujarse con el declive de este paradigma y que dan entrada a los discursos sobre la precariedad ya estaban presentes en las formas laborales femeninas del anterior tiempo.

2. Castro (2008) se basa en el trabajo de Ramos y Prieto (1999), que argumentan que son tres los aspectos fundamentales ligados a los tiempos de trabajo: (a) el tiempo de relación contractual (o contratos), (b) el tiempo de prestación laboral (horarios y calendarios) y (c) el tiempo de la vida laboral (o trayectoria profesional). Castro (2008), ampliando este espectro temporal, añade un cuarto elemento de análisis: las expectativas laborales. El autor defiende que, como fruto de las transformaciones de los tiempos de trabajo, no solo se ha dado una diversificación de las relaciones contractuales, de las jornadas laborales o de las biografías laborales, sino también de las expectativas laborales de los trabajadores. Según su argumentación, los individuos, con el fin de dotar de orden a sus vidas, se esfuerzan por establecer ciertos niveles de continuidad y coherencia entre su pasado, su presente y su futuro. Los planes de vida o proyectos vitales, articulados a través de decisiones, forman parte de las diversas estrategias que despliegan los individuos para lograr este objetivo. Así, planes, proyectos y decisiones quedan unidos a las expectativas biográficas.
3. El hecho de que las guías vitales hayan perdido una gran parte de su capacidad para generar pautas de ordenamiento y que las opciones por las que avanzar se multipliquen provoca que el proceso de elección individual tome una especial relevancia en los análisis del ciclo vital.

4. A pesar de que el género es una variable que se ha tenido en cuenta a la hora de llevar a cabo la investigación, no se extraen elementos significativos que haya que incluir en los temas que aquí se trabajan.

5. Es importante recordar que estos resultados se circunscriben al perfil descrito en el primer apartado del artículo y no a la población joven en general. 


\section{BIBLIOGRAFÍA}

Alonso, L. E. (1999). Trabajo y ciudadanía. Estudios sobre la crisis de la sociedad salarial. Madrid: Trotta.

Alonso, L. E. (2007). La crisis de la ciudadanía laboral. Barcelona: Anthropos.

Artegui Alcaide, I. (2014). La continuidad biográfica y el manejo de la incertidumbre: análisis de la realidad transicional de los jóvenes adultos. En: Araujo, E., Duque, E., Franch, M. y Durán, J. (eds.). Tempos Sociais e o Mundo Contemporãneo. As crises, as fases e as ruturas. Universidade do Minho: Centro de Estudos de Comunicação e Sociedade, pp. 7-20.

Beck, U. (1992). La sociedad del riesgo. En camino hacia otra sociedad moderna. Barcelona: Paidós.

Beck, U. y Beck-Gernsheim, E. (2003). La individualización. El individualismo institucionalizado y sus consecuencias sociales y políticas. Barcelona: Paidós.

Bernardi, F. (2007). Movilidad social y dinámicas familiares. Una aplicación al estudio de la emancipación familiar en España. Revista Internacional de Sociología, LXV, 48, pp. 33-54.

Bradley, H.y Devadason, R. (2008). Fractured Transitions: Young Adults' Pathways into Contemporary Labour Markets. Sociology, 42, 1, pp. 119-136. https://doi. org/10.1177/0038038507084828

Brannen, J. y Nilsen, A. (2002). Young people's time perspectives. From youth to adulthood. Sociology, 36, 3, pp. 513-537. https:// doi.org/10.1177/0038038502036003002

Carrasquer, P. y Torns, T. (2007). Cultura de la precariedad: conceptualización, pautas y dimensiones. Una aproximación desde la perspectiva de género. Sociedad y Utopía. Revista de Ciencias Sociales, 29, pp. 139-156.

Castro, C. de (2008). La influencia de las expectativas en la organización temporal de la vida laboral. Política y sociedad, 45, 2, pp. 169-188.

Cavalli, A. (1985). Il tempo dei giovani. Bologna: II Mulino.

Chisholm, L., Brown, P., Bucher, P. y Kruger, H. H. (1990). Chilhood and youth studies in the United Kingdom and West Germany: an introduction. En: Chisholm, L. et al (eds.) Childhood, youth and social change. A comparative perspective. London: Farmer.
Côté, J. E. (2000). Arrested Adulthood. The Changing Nature of Maturity and Identity. Nueva York y Londres: New York University Press.

Devadason, R. (2008). To Plan or Not to Plan? Young Adult Future Orientations in Two European Cities. Sociology, 42, 6, pp. 1127-1145. https://doi. org/10.1177/0038038508096937

du Bois-Reymond, M. (1998). 'I don't want to commit myself yet': Young people's life concepts. Journal of Youth Studies, 1, 1, pp. 63-79. https://doi.org/10.1080 /13676261.1998.10592995

du Bois-Reymond, M. y López Blasco, A. (2004). Transiciones tipo yo-yo y trayectorias fallidas: hacia las políticas integradas de transición para los jóvenes europeos. Revista de Estudios de Juventud, 65, pp.11-29.

Elchardus, M. y Smitts, W. (2006). The Persistence of the Standardized Life Cycle. Time and Society, 15, 2, pp. 303-326. https:// doi.org/10.1177/0961463X06066944

Fenton, S. y Dermott, E. (2006). Fragmented careers? Winners and losers in young adult labour markets. Work, Employment \& Society, 20, 2, pp. 205-221. https://doi. org/10.1177/0950017006064111

Furlong, A. y Cartmel, F. (1997). Young people and social change. Individualization and risk in late modernity. Buckingham: Open University Press.

Furlong, A., Cartmel, F. y Biggart, A. (2006). Choice biographies and transitional linearity. Re-conceptualising modern youth transitions. Papers, 79, pp. 225 - 239. https://doi.org/10.5565/rev/papers/v79n0.834

Gentile, A. (2014). Inestabilidad laboral y estrategias de emancipación. Una tipología de jóvenes-adultos mileuristas. Acciones e Investigaciones Sociales, 34, pp. 125-154.

Kohli, M. y Rein, M. (1991). Time for Retirement. Cambridge: Cambridge University Press.

Leccardi, C. (2002). Tiempo y construcción biográfica en la "sociedad de la incertidumbre": reflexiones sobre las mujeres jóvenes. Nómadas, 16, pp. 42-50.

Leccardi, C. (2005). Facing uncertainty. Temporality and biographies in the new century. Young - Nordic Journal of Youth Research, 13, 2, pp. 123-146.
Leccardi, C. (2008). New Biographies in the "Risk Society"? About Future and Planning. 21st Century Society, 3, 2, pp. 119-129. https://doi. org/10.1080/17450140802062078

Leccardi, C. y Ruspini, E. (eds.) (2006). A New Youth? Young People, Generations and Family Life. Aldershot: Ashgate.

Luhmann, N. (1976). The Future Cannot Begin: Temporal Structures in Modern Society. Social Research, 43, pp. 130-152 [trad. en Ramos, R. (comp.) (1992). Tiempo y sociedad. Madrid: Centro de Investigaciones Sociológicas, pp.161-182].

Moreno Mínguez, A. (coord.), López Peláez, A. y Segado Sánchez-Cabezudo, S. (2012). La transición de los jóvenes a la vida adulta. Crisis económica y emancipación tardía. Barcelona: Obra Social La Caixa.

Nilsen, A. (1999). Where Is the Future? Time and Space as Categories in Analyses of Young People's Images of the Future. Innovation, 12, 2, pp. 175-194. https://doi.org/10.1080/13511610.199 9.9968596

Pais, J. M. (2003). The Multiple Faces of the Future in the Labyrinth of Life, Journal of Youth Studies, 6, 2, pp. 115-126. https:// doi.org/10.1080/1367626032000110264

Prieto, C. (2007). Del estudio del empleo como norma social al de la sociedad como orden social. Papeles del CEIC. International Journal on Collective Identity Research, 1, papel 28. Disponible en: http://www.ehu.eus/ojs/index.php/ papelesCEIC/article/view/12197/11119

Piore, M. J. y Sabel, C. F. (1990). La segunda ruptura industrial. Madrid: Alianza.

Ramos Torre, R. y Prieto Rodríguez, C. (1999). El tiempo de trabajo: entre la competitividad y los tiempos sociales. En: Prieto Rodríguez, C. y Miguélez Lobo, F. (1999). Las relaciones de empleo en España. Madrid: Siglo XXI, pp. 463-488.

Reiter, H. (2003). Past, Present, Future: Biographical Time Structuring of Disadvantaged Young People. Young, 11, 3, pp. 253-279. https://doi. org/10.1177/11033088030113004

Santamaría, E. (2010). "Buscarse la vida": trayectorias y experiencias de precariedad en el acceso al empleo de las personas jóvenes. Revista de Estudios de Juventud, 89, pp. 101-123. 
Santamaría, E. (2012). Jóvenes y precariedad laboral. Trayectorias laborales por los márgenes del empleo. Zerbitzuan, 52, pp. 129-139. https://doi.org/10.5569/11347147.52 .08

Santos, J. A. y Martín, M. P. (2012). La juventud española en tiempos de crisis. Paro, vidas precarias y acción colectiva. Sociología del Trabajo, 75, pp. 93-110.
Stauber, B. y Walther, A. (2006). De-standardised pathways to adulthood: European perspectives on informal learning in informal networks. Papers, 79, pp. 241 - 262. https://doi.org/10.5565/rev/ papers/v79n0.835

Torns, T. y Recio Cáceres, C. (2012). Las desigualdades de género en el mercado de trabajo: entre la continuidad y la trans- formación. Revista de Economía Crítica, 14, segundo semestre, pp. 178-202.

Wilkinson, H. (1997). Hijos de la libertad. ¿Surge una nueva ética de la responsabilidad individual y social? En: Beck, U. (coord.) (1997) Hijos de la libertad. México: Fondo de Cultura Económica, pp. 87-128. 